\title{
Structure and Dynamics of Loosely Cross-Linked Ionic Microgel Dispersions in the Fluid Regime
}

\author{
P. Holmqvist, ${ }^{1, *}$ P. S. Mohanty, ${ }^{2, \dagger}$ G. Nägele, ${ }^{1}$ P. Schurtenberger, ${ }^{2}$ and M. Heinen ${ }^{3}$ \\ ${ }^{1}$ Institute of Complex Systems (ICS-3), Forschungszentrum Jülich, Jülich D-52425, Germany \\ ${ }^{2}$ Division of Physical Chemistry, Lund University, Lund SE-221 00, Sweden \\ ${ }^{3}$ Institut für Theoretische Physik II, Weiche Materie, Heinrich-Heine-Universität Düsseldorf, Düsseldorf D-40225, Germany
}

(Received 3 February 2012; published 26 July 2012)

\begin{abstract}
We report a comprehensive experimental-theoretical study of the temperature- and concentrationdependent swelling behavior of weakly cross-linked PNiPAm ionic microgel particles in the deionized fluid phase. The particles swell reversibly when the dispersion is cooled from the collapsed state to lower temperatures. While the collapsed state shows no dependence on the microgel number density, the swelling at lower $T$ is more pronounced at lower concentrations. The static pair correlations and shorttime diffusion functions, and the concentration and temperature dependence of the microgel radius and effective charge, are studied using static and dynamic light scattering in combination with state-of-the-art analytical theoretical schemes based on a Yukawa-type effective pair potential and a core-shell model. We show that only such a combined, simultaneous fit of static and dynamic scattering functions allows for an unambiguous determination of the microgel radius and effective charge.
\end{abstract}

DOI: 10.1103/PhysRevLett.109.048302

PACS numbers: 82.70.Dd, 78.35.+c

Dispersions of cross-linked microgel particles such as poly ( $N$-isopropylacrylamide) (PNiPAm) $[1-5]$ have become popular model systems of soft colloids. The most prominent feature of these systems is their stimulus sensitivity. They can undergo phase transitions, as the particle volume reversibly responds to environmental stimuli such as $p \mathrm{H}$ value, temperature, hydrostatic pressure, ionic strength, or an external electromagnetic field [6]. These features, and the biocompatibility of the microgels, allow for their use in biomedical applications [7]. The physical nature of the microgel particles lies in between that of hard-sphere colloids, and ultrasoft star polymers and micelles [2,8]. Because of the repulsive soft pair interactions, microgels can interpenetrate or compress. Hence, phases with number densities above solid-sphere close packing can be prepared, exhibiting peculiar structural and dynamic properties $[9,10]$. The precise form of the microgel pair interaction potential is currently unknown. The studies of PNiPAm dispersions carried out so far dealt mostly with their microstructure, and the concentrationand temperature dependent phase behavior in the highly concentrated state $[1-5,10,11]$.

Microgels such as the ones in the present study, which are copolymerized with ionic groups, attain a nonzero effective charge in a polar solvent and are referred to as ionic microgels [12]. They belong to the subclass of ultrasoft colloids where the effective pair potential crosses over from a screened Coulomb (Yukawa) type repulsion at nonoverlap distances to a smoothly increasing repulsion interaction energy at overlap. In earlier experiments [12], a variety of phases including fluid, crystalline, and glassy states of ionic microgels at different concentrations were investigated. A fraction of the counterions released by ionic polymer backbone groups is confined to the interior of the microgel particles. The electric repulsion between polyelectrolyte chain segments, and the osmotic pressure by the counterions can lead to a pronounced swelling of the microgels as compared to electrically neutral ones [10,13-15]. Temperature changes can significantly change the solvent-polymer interactions and the microionic osmotic pressure, hence affecting the outer (effective) hydrodynamic radius, $R_{\text {tot }}$, and the effective charge, $Z e$, with $e$ the proton elementary charge.

At fixed microgel number density, $n_{p}$, even small changes in $R_{\text {tot }}$ can cause dramatic changes in the microgel volume fraction $\varphi=(4 \pi / 3) R_{\mathrm{tot}}{ }^{3} n_{p}$. A common approach to determine $\varphi$ is to extract $R_{\mathrm{tot}}$ as a function of temperature, $T$, in the dilute regime of noninteracting particles from measuring the form factor, $P(q)$, single-particle diffusion coefficient or intrinsic viscosity, and to calculate $\varphi$ at low as well as at high values of $n_{p}$ from this "known" size $[5,12,16]$. This approach neglects a possible dependence of $R_{\mathrm{tot}}$ on $n_{p}$ which, as we are going to show, is quite significant. Studies on the structure and dynamics of neutral PNiPAm particles have been reported in $[4,5,9]$, where very high $\varphi$ were considered, even above solid-sphere random close packing. The present study differs in that the microgels are charged and weakly cross-linked. Therefore, a significant $n_{p}$ dependence of the thermoresponsive swelling can be expected at lower $\varphi$, including the fluid regime. To our knowledge, no experimentaltheoretical analysis comparable to the present one has been carried out for the fluid phase of weakly cross-linked ionic microgels.

The 1.55 mol-\% poly(N-isopropylacrylamide)-co-poly (acrylic acid) (PNiPAm-co-PAA) microgels are dispersed in deionized water at temperatures between 15 and $52^{\circ} \mathrm{C}$. Based on literature $[5,13,17,18]$, the mean mesh size lies in 
between 5 and $10 \mathrm{~nm}$. Microgel dispersions of various concentrations were prepared in $5 \mathrm{~mm}$ NMR cells, together with a mixed-bed ion exchange resin (Biorad, USA), and then flame sealed. This procedure ensures a constant low salinity. Our DLS measurements were performed using a setup by ALV Laservertriebsgesellschaft, Germany. The normalized intensity time autocorrelation function $g_{2}(q, t)=\langle I(q, 0) I(q, t)\rangle /\langle I(q, 0)\rangle^{2}$ was recorded by an ALV-5000 multitau digital correlator. The microgel particles are loosely cross-linked, with a very low polymer volume fraction of 0.005 .

Figure 1 shows $g_{2}\left(q_{\max }, t\right)$ at $n_{p}=0.0053 \mu \mathrm{M}$ and $n_{p}=0.022 \mu \mathrm{M}$, for two different $T$. Here, $q_{\max }$ is the wave number at which the principal maximum in the structure factor, $S(q)$, occurs. The time axis is made dimensionless by the characteristic time $\tau=$ $6 \pi \eta(T) R_{\text {tot }}\left(52^{\circ} \mathrm{C}\right) /\left(q_{\max }{ }^{2} k_{B} T\right)$ related to the collapsed particle radius $R_{\text {tot }}$ at $T=52{ }^{\circ} \mathrm{C}$, with Boltzmann constant $k_{B}$, and viscosity, $\eta(T)$, of water. It is clear from Fig. 1 that at $T=52{ }^{\circ} \mathrm{C}$, the $g_{2}\left(q_{\max }, t\right)$ at both $n_{p}$ values coincide practically. At $T=34{ }^{\circ} \mathrm{C}, g_{2}\left(q_{\max }, t\right)$ is different for different $n_{p}$. This suggests that even in the fluid phase, the radius $R_{\text {tot }}$ of swollen microgels, and hence the (cage) diffusion coefficient, $D\left(q_{\max }\right)$, depend strongly on $n_{p}$ at lower $T$.

To gain more insight into the swelling behavior, we have measured $I(q)$ and the short-time diffusion function, $D(q)$, for five microgel concentrations from 0.0053 to $0.069 \mu \mathrm{M}$ over the full accessible $q$ range, and at various $T$ from $15-52{ }^{\circ} \mathrm{C}$. To extract $D(q)$ from $g_{2}(q, t)$ the latter was analyzed as described in [19].

In Fig. 2 and 3, $D(q)$ and the normalized experimental intensity, $I(q) / I_{0}$, respectively, are shown. Here, $I_{0}$ is the laser source intensity corrected for scattering volume. Note that we have disregarded microgel permeability effects on $D(q)$ and $D_{0}$, with the latter defined by $D_{0}\left(T, n_{p}\right)=$ $k_{B} T /\left(6 \pi \eta(T) R_{\text {tot }}\left(T, n_{p}\right)\right)$. Figures 2 and 3 comprise data for $n_{p}=0.0053 \mu \mathrm{M}$ and $n_{p}=0.022 \mu \mathrm{M}$. In the two

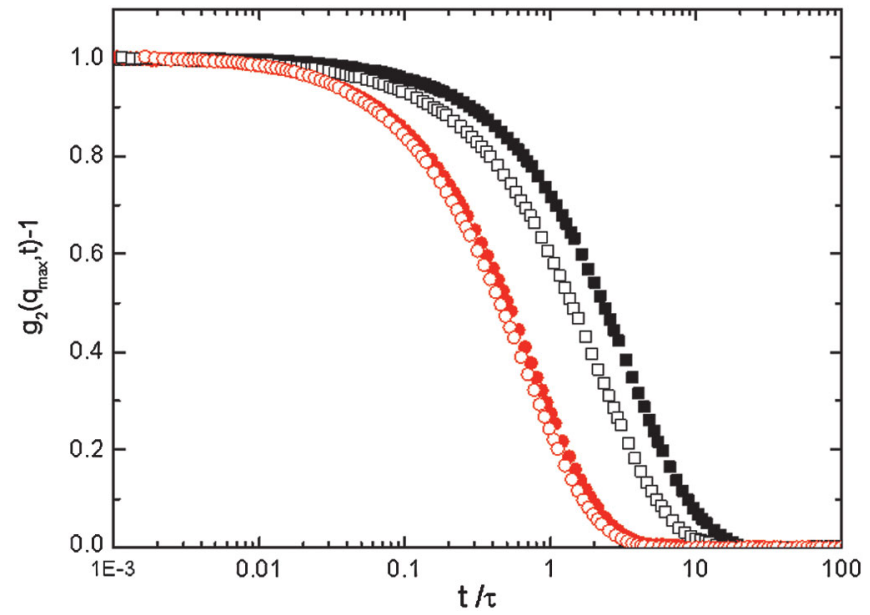

FIG. 1 (color). $\quad g_{2}\left(q_{\max }, t\right)-1$, for $n_{p}=0.0053 \mu \mathrm{M}$ (filled symbols) and $n_{p}=0.022 \mu \mathrm{M}$ (open symbols), at $q=q_{\max }$ for $T=34{ }^{\circ} \mathrm{C}$ (squares) and $52{ }^{\circ} \mathrm{C}$ (circles). series of Fig. 2, it is observed that the principal minimum in $D(q)$ at $q_{\max }$ deepens with decreasing $T$ at fixed $n_{p}$, indicative of increasing interaction strength. Similarly, $D\left(q_{\mathrm{max}}\right)$ becomes smaller with increasing $n_{p}$ at fixed $T$, and the principal peak in $I(q)$, Fig. 3 , at $q_{\max }$ becomes more pronounced with increasing $n_{p}$ or decreasing $T$. We checked that $q_{\max }$ scales as $n_{p}{ }^{1 / 3}$, which is the concentration scaling for charged colloids at low salinity [20,21]. For an analytic treatment, we model the pair potential as the HSY repulsive part of the Derjaguin-Landau-Verwey-Overbeek potential $[20,21]$. According to $[7,10,11]$, a screened Coulomb potential describes the electrostatic repulsion of ionic microgels at nonoverlap distances. For the considered concentration regime, where $\kappa R_{\text {tot }} \leq 1$, only a small fraction of mobile counterions are penetrating the region $R<R_{\text {tot }}$ while not being condensed on the polymer backbones [12,22]. The HSY potential agrees thus practically with the nonoverlap part of the effective pair potential for weakly cross-linked, uniform ionic microgels derived by Denton using linear response theory [22]. Here, $\kappa$ is the Debye screening parameter determined by the mobile counterions released by the polymer network, and by the $\mathrm{H}^{+}$and $\mathrm{OH}^{-}$ions from the autoproteolysis of water. Since the samples were flame-sealed together with ion exchange resin, we assume the concentration of additional, parasitic salt ions to be zero. Under this proviso, $\kappa$ is fully determined by $n_{p}, Z$, and the concentration, $c_{\mathrm{OH}^{-}}$, of negative coions calculated using tabulated, $T$-dependent $\mathrm{pKw}$ values of water. The quantity, $Z$, is the bare charge number corrected for counterions which are condensed on the polyelectrolyte chains or at least constrained to move along the chains, minus the number of mobile counterions inside the microgel sphere. It includes also a contribution from the electrostatically strongly coupled counterions near the outer surface. In a conductivity titration and light scattering study in [12], the number of dissociable groups, $Z_{\text {bare }}<$ $3.7 \times 10^{4}$, has been determined for PNIPAM-co-PAA

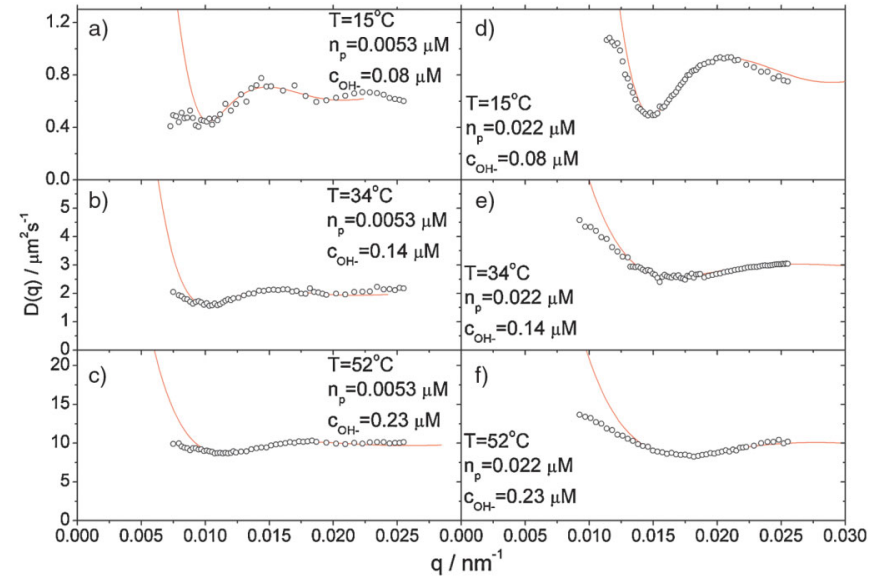

FIG. 2 (color). $D(q)$ for $n_{p}=0.0053 \mu \mathrm{M}$ (left panels) and $n_{p}=0.022 \mu \mathrm{M}$ (right panels), and for $T$ as indicated. Lines are fit results for $D(q)$. 


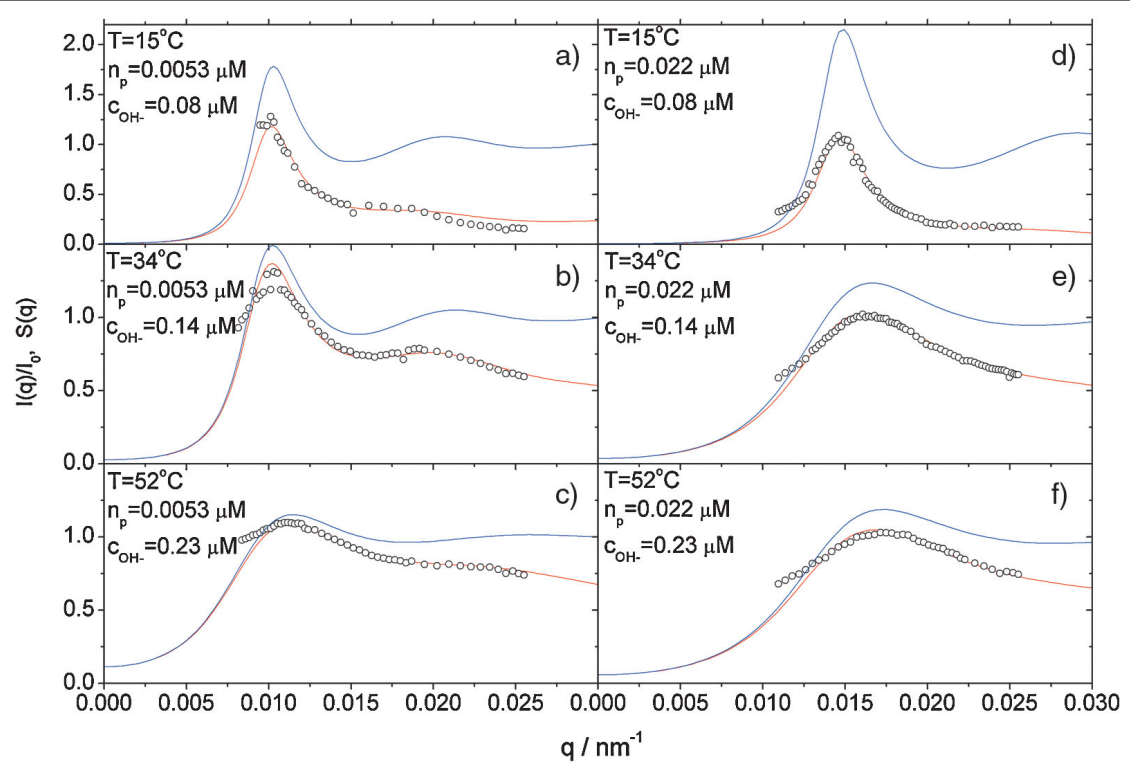

FIG. 3 (color). $I(q) / I_{0}$ for $n_{p}=0.0053 \mu \mathrm{M}$ (left panels) and $n_{p}=0.022 \mu \mathrm{M}$ (right panels), and for $T$ as indicated. Red lines are fit results for $I(q) / I_{0}=P(q) S(q)$ and blue lines are $S(q)$ computed by the MPB-RMSA.

particles of sizes similar to the present ones. Fits of static light scattering (SLS) data in [12] gave $Z \sim 300$, consistent with our present findings. The 2 orders of magnitude difference between $Z_{\text {bare }}$ and $Z$ indicates that the vast majority of the interior counterions are condensed or at least one-dimensionally constrained on the polyelectrolyte chains, while only a small fraction is free to move three dimensionally in between the polymer chains.

Using the hard-sphere plus Yukawa (HSY) potential dependent on $\varphi, Z$, and $R_{\text {tot }}$, we have calculated $S(q)$ by the modified penetrating background corrected rescaled mean spherical approximation (MPB-RMSA) scheme $[20,21]$. The so-obtained $S(q)$ was used as input for calculating $D(q) / D_{0}$ by the modified $\delta \gamma$ scheme in [23,24] which accounts for many-body hydrodynamic interactions. The modification amounts to replacing the self-diffusion coefficient part, $D_{s}=D(q \rightarrow \infty)$, of $D(q)$ by a more accurate expression obtained assuming pairwise additive hydrodynamic interactions [24]. The high accuracy of the MPB-RMSA for $S(q)$, and the modified $\delta \gamma$ scheme for $D(q)$, were validated in comparison to experimental data and elaborate simulations [20,21,24-27]. As a consequence, each of the fit parameters $\varphi, Z$, and $R_{\text {tot }}$ is obtained to accuracy better than $5 \%$. Here, we take advantage of the predictive power of these analytic schemes to determine the size and effective charge of the microgel particles. The fit parameters $n_{p}, Z$, and $R_{\text {tot }}$ enter into the calculations both of $D(q) / D_{0}$ and $I(q) / I_{0}=P(q) S(q)$, with $n_{p}$ taken as a global fit parameter for each considered concentration. To minimize the number of parameters, we model $P(q)$ by a simple core-shell model [28]. In small-angle neutron scattering studies [29,30], it was found that the polymer density is practically constant in the dense core region, surrounded by a shell of reduced polymer density. We assume an isotropic relative optical permittivity, $\left[\varepsilon_{p}-\varepsilon_{s}\right] / \varepsilon_{s}$, where $\varepsilon_{p}$ and $\varepsilon_{s}$ are the permittivities of particle and solvent, respectively. The radial dependence of $\varepsilon_{p}$ is modeled by a step function, i.e., $\varepsilon_{p}\left(R<R_{\text {cor }}\right)=\varepsilon_{1}$ and $\varepsilon_{p}\left(R_{\text {cor }}<R<R_{\text {tot }}\right)=\varepsilon_{2}$. In the optical frequency range of our static and dynamic light scattering (SLS/DLS) experiments, the permittivity of PNiPAm exceeds that of water, so that $\varepsilon_{1}>\varepsilon_{2}$ [31]. In the fully collapsed state where $T>52^{\circ} \mathrm{C}$, the core-shell structure reduces to a homogeneous sphere, with $R_{\text {cor }}$ and $R_{\text {tot }}$ being identical. Since $\varepsilon_{1}, \varepsilon_{2}, R_{\text {cor }}$, and $R_{\text {tot }}$ depend in principle both on $T$ and $n_{p}$, they cannot be uniquely determined simply from fitting $P(q)$ at low $n_{p}$. When $n_{p}$ and $T$ are varied, a complication arises from the necessity to determine $Z\left(T, n_{p}\right)$, which enters the calculation of $S(q)$, in addition to $R_{\mathrm{tot}}\left(T, n_{p}\right)$. To cope with this, we simultaneously analyze both the SLS scattered intensity $I(q)=$ $I_{0} P(q) S(q)$, and $D(q)$. This analysis has become possible with the advent of the fast MPB-RMSA and modified $\delta \gamma$ schemes for calculating $S(q)$ and $D(q)$.

Figure 2 exemplifies our best-fit results for $D(q)$ and Fig. 3 for $I(q) / I_{0}$. The high quality of the fits is maintained for all investigated samples. The scatter in our SLS/DLS data depends on the scattering-signal to background ratio and, in particular, on the optical-permittivity contrast between particles and solvent. The largest relative scatter of about $10 \%$ is found for the dilute samples of maximum swollen particles in panel (a) of Fig. 2 and Fig. 3, panel (a). Systematic deviations between theoretical and experimental results are observable at small $q$ only, and are in line with an intensity upturn frequently reported in many charged-particle systems at low salinity when $q \ll q_{\max }$ $[32,33]$. The origin of this upturn is largely unknown. For our microgel system, van der Waals attraction, polydispersity and residual multiple scattering are too weak to cause 
this effect. Since this upturn has no bearing on our main conclusions, it will not be considered any further.

In Fig. 4(a), results for $R_{\text {tot }}$ are plotted as functions of $T$, for all investigated $n_{p}$. As one might expect, the particles radius in the high- $T$ collapsed state does not depend on $n_{p}$. In the investigated $T$-range, the fully collapsed state is not reached by the very dilute samples of noninteracting particles at $n_{p}=0.1 \mathrm{nM}$, and at a $p \mathrm{H}$-value of 6.3 (open squares). However, the collapsed state is reached, when the particles are charge-neutralized by reducing the $p \mathrm{H}$ to 3.0 (open circles). The $R_{\mathrm{tot}}(T)$ for the two dilute systems exhibit thermo-sensitive swelling behavior at very different transition temperatures. This is a known feature, attributed to the $p \mathrm{H}$-dependent charge content inside a PNiPAm-coPAA particle owing to the deprotonation of the carboxylic groups with increasing $p \mathrm{H}$ value [34]. A detailed discussion of $p \mathrm{H}$-dependent swelling of PNiPAm-co-PAA microgels is given in [12]. For more concentrated dispersions of interacting particles at neutral $p \mathrm{H}$ value (filled symbols) the $R_{\mathrm{tot}}(T)$ swelling curves are bound from above and below by those of the dilute systems at measured $p \mathrm{H}=6.3$ and 3.0, respectively. The swelling transition of the interacting particle samples is systematically

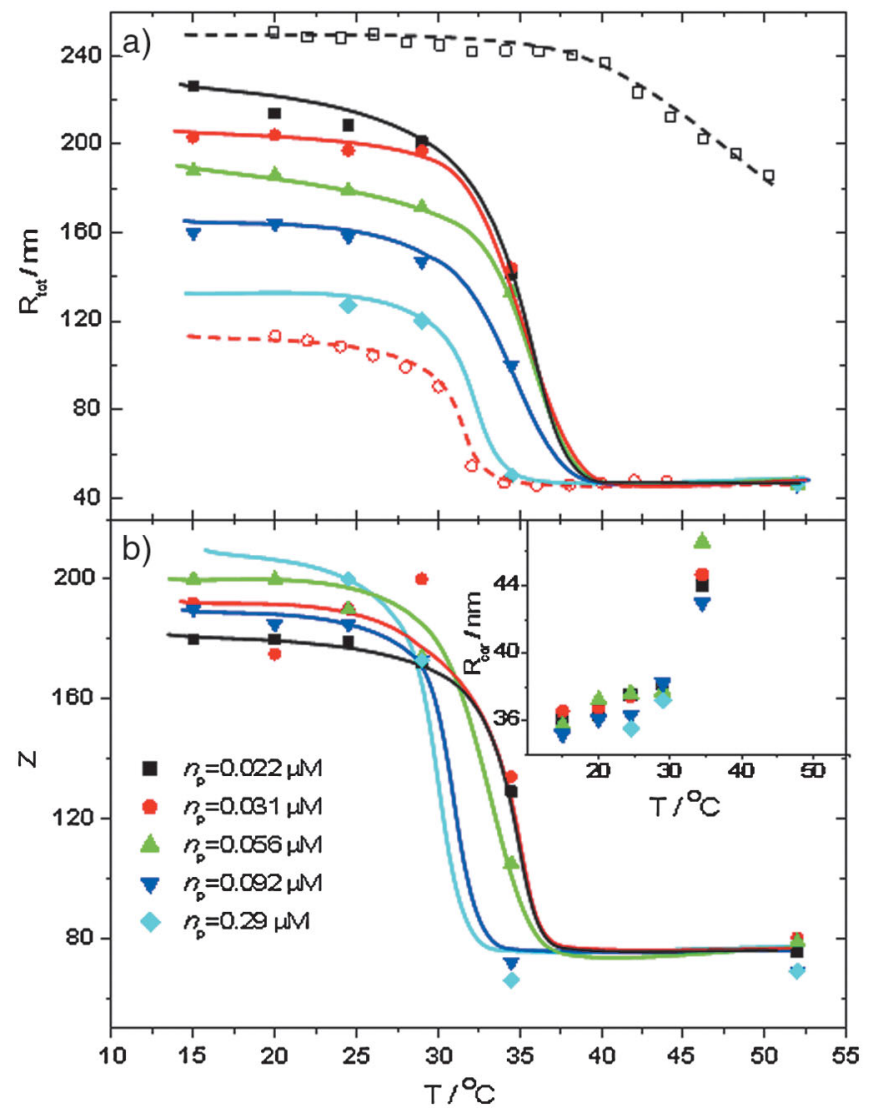

FIG. 4 (color). (a) $R_{\text {tot }}$ and (b) $Z$ as a function of $T$, for different $n_{p}$ as indicated. Open symbols in (a): Results for dilute noninteracting particles with $n_{p}=0.1 \mathrm{nM}$ and $p \mathrm{H}=6.3$ (black), and $p \mathrm{H}=3.0$ (red). Inset in (b): $R_{\text {cor }}$ as a function of $T$. Lines are guides to the eyes. shifting to lower $T$ with increasing $n_{p}$. Additionally, the swollen-state radius, $R_{\text {tot }}$, at low $T$ reduces substantially with increasing $n_{p}$. The observed shift in the transition temperature exceeds $5{ }^{\circ} \mathrm{C}$, and $R_{\text {tot }}$ of the swollen state, at given $T$, varies by about $50 \%$.

We discuss next the behavior of $Z$ as obtained from our combined fits of $I(q)$ and $D(q)$. In Fig. 4(b), $Z$ is displayed as a function of $T$ for various $n_{p}$. At higher $T$, i.e., in the collapsed state, $Z$ attains a value of about 70 , independent of $n_{p}$. As $T$ is lowered, the particles swell and $Z$ increases strongly to values of about 170-200. Moreover, as $n_{p}$ is increased at a fixed $T$ in the swollen state, $Z$ increases slightly whereas $R_{\text {tot }}$ decreases. The effective charge density of a swollen-state microgel therefore increases with increasing $n_{p}$. Note from the inset of Fig. 4(b) that within experimental scatter, $R_{\text {cor }}(T)$ does not depend on $n_{p}$, except for $T$ close to the transition temperature. This implies that while a change in $T$ affects a particle as a whole, concentration changes influence the shell swelling only.

We argue that the $n_{p}$-dependent swelling of loosely cross-linked ionic microgels is to a large extent a microionic osmotic pressure effect: An increase in $n_{p}$ enlarges the concentration of counterions outside the microgels. For the present microgels, the total ionic strength, obtained from our theoretical analysis, changes by a factor of up to ten in going from the lowest to the highest concentration. Since the counterion osmotic pressure imbalance counteracts the swelling of particles, both the swollen-state outer radius and the transition temperature are lowered with increasing concentration. A similar shift in the transition temperature was also reported for dispersions of nonionic PNiPAm microgels, where the concentration of added salt was the control parameter [35]. Although the bulk $p \mathrm{H}$ is kept at about 7.0, with increasing $n_{p}$ it is possible that there is some charge regulation between shell and bulk, influenced also by the Coulombic gel-gel interaction. This can affect the local $p \mathrm{H}$ conditions inside the particles, which in turn can influence not only the osmotic pressure, but also the polymer-solvent parameter. A slight increase in this parameter could reduce the size of the particle at a fixed effective charge. A change in particle size with increasing $n_{p}$ is therefore reasonable, even for (nearly) constant $Z$. However, a thorough understanding of the insensitivity of $Z$ in Fig. 4(b) regarding the gel size has to await a theory of the swelling of interacting core-shell type ionic microgels.

Swollen microgels exhibit some solvent permeability, which affects $D_{0}$, and to a lesser extent also $D(q) / D_{0}$ $[36,37]$. The effect on $D_{0}$ can be computed analytically on basis of a Brinkmann model of solvent flow inside the particles resulting in an increase in $D_{0}$ of less than $20 \%$. For charged permeable particles at moderately small $\varphi$, we have estimated the permeability influence on $D(q) / D_{0}$ [24,37] and found that permeability affects $D(q) / D_{0}$ considerably less in ionic than in nonionic systems. Therefore, we refrain from including the small permeability effects into our dynamic calculations. Moreover, the 
current knowledge of the hydrodynamic structure of microgels is not detailed enough for an ab-initio calculation of the $R_{\mathrm{tot}}$-dependent particle permeability.

The importance of our experimental-theoretical results derives from the fact that microgel dispersions are a popular and widely studied system in soft matter science, for which a precise determination of $\varphi$ and $Z$ is essential. We have shown that simply measuring the behavior of $R_{\mathrm{tot}}(T)$ under dilute conditions can lead to the prediction of particle volume fractions in concentrated systems which differ from the true values by a factor of up to eight. For example, the transition concentration at a fluid-crystal or glass transition point could be easily misinterpreted, with results in apparent conflict with the predictions by theory and simulation. In addition, our results for $R_{\text {tot }}\left(T, n_{p}\right)$ and $Z\left(T, n_{p}\right)$ can be useful for a comparison with predictions by theories or simulations of the swelling and coil-to-globule transition of interacting microgels. Our results on the $n_{p}$ dependence of $R_{\text {tot }}$ are relevant also to the filtration of ionic microgel suspensions, and pressure-driven microgel translocation through pores [38].

The microgel systems studied here constitute in a sense an extreme case, since both the cross-link density and salinity are very low. However, even under less extreme conditions, a concentration-dependent swelling can have dramatic consequences, since a $10 \%$ change in $R_{\text {tot }}$ corresponds to a $25 \%$ change in $\varphi$. As $P(q), S(q), D(q)$ and $D_{0}$ are all $n_{p}$ and $T$ dependent in ionic microgel dispersions, only a simultaneous fit of static and dynamic properties ensures consistency and reliability of the fit parameters. With the fast and accurate MPB-RMSA and the modified $\delta \gamma$ scheme for $S(q)$ and $D(q)$ available, we have the theoretical tools for this simultaneous fitting, and for a detailed analysis of pair correlations and short-time diffusion properties in ionic microgels.

We thank Simon Rogers (Research Centre Jülich) for carefully proofreading the letter. P. S. M. and P. S. acknowledge financial support from the Swiss National Science Foundation. G. N. and M.H. acknowledge support from the Deutsche Forschungsgemeinschaft (project B2 in SFB-TR6).

*p.holmqvist@fz-juelich.de †priti.mohanty@fkem1.lu.se

[1] T. Eckert and W. Richtering, J. Chem. Phys. 129, 124902 (2008).

[2] D. M. Heyes and A. C. Branka, Soft Matter 5, 2681 (2009).

[3] S. Pyett and W. Richtering, J. Chem. Phys. 122, 034709 (2005).

[4] F. Scheffold, P. Diaz-Leyva, M. Reufer, N. Ben Braham, I. Lynch, and J. L. Harden, Phys. Rev. Lett. 104, 128304 (2010).

[5] M. Stieger, J. S. Pedersen, P. Lindner, and W. Richtering, Langmuir 20, 7283 (2004).

[6] M. Das, H. Zhang, and E. Kumacheva, Annu. Rev. Mater. Res. 36, 117 (2006).
[7] Y. Guan and Y. J. Zhang, Soft Matter 7, 6375 (2011).

[8] L. A. Lyon and A. Fernandez-Nieves, Annu. Rev. Phys. Chem. 63, 25 (2012).

[9] D. A. Sessoms, I. Bischofberger, L. Cipelletti, and V. Trappe, Phil. Trans. R. Soc. A 367, 5013 (2009).

[10] C. N. Likos, in Microgel Suspensions: Fundamentals and Applications, edited by A. Fernandez-Nieves et al. (WileyVCH Verlag GmbH \& Co. KGaA, Weinheim, 2011).

[11] D. Gottwald, C. N. Likos, G. Kahl, and H. Lowen, Phys. Rev. Lett. 92, 068301 (2004).

[12] P. S. Mohanty and W. Richtering, J. Phys. Chem. B 112, 14692 (2008).

[13] A. Fernandez-Barbero, A. Fernandez-Nieves, I. Grillo, and E. Lopez-Cabarcos, Phys. Rev. E 66, 051803 (2002).

[14] Y. Levin, A. Diehl, A. Fernandez-Nieves, and A. Fernandez-Barbero, Phys. Rev. E 65, 036143 (2002).

[15] M. Quesada-Perez, J. A. Maroto-Centeno, J. Forcada, and R. Hidalgo-Alvarez, Soft Matter 7, 10536 (2011).

[16] H. Senff and W. Richtering, J. Chem. Phys. 111, 1705 (1999).

[17] K. Kratz, T. Hellweg, and W. Eimer, Polymer 42, 6631 (2001).

[18] K. Kratz, A. Lapp, W. Eimer, and T. Hellweg, Colloids Surfaces A 197, 55 (2002).

[19] P. Holmqvist and G. Nägele, Phys. Rev. Lett. 104, 058301 (2010).

[20] M. Heinen, P. Holmqvist, A. J. Banchio, and G. Nägele, J. Chem. Phys. 134, 044532 (2011).

[21] M. Heinen, P. Holmqvist, A. J. Banchio, and G. Nägele, J. Chem. Phys. 134, 129901 (2011).

[22] A. R. Denton, Phys. Rev. E 67, 011804 (2003).

[23] C. W. J. Beenakker and P. Mazur, Physica (Amsterdam) 126A, 349 (1984).

[24] M. Heinen, A. J. Banchio, and G. Nägele, J. Chem. Phys. 135, 154504 (2011).

[25] A. J. Banchio, J. Gapinski, A. Patkowski, W. Haussler, A. Fluerasu, S. Sacanna, P. Holmqvist, G. Meier, M. P. Lettinga, and G. Nägele, Phys. Rev. Lett. 96, 138303 (2006).

[26] A. J. Banchio and G. Nägele, J. Chem. Phys. 128, 104903 (2008).

[27] M. Heinen, P. Holmqvist, A. J. Banchio, and G. Nägele, J. Appl. Crystallogr. 43, 970 (2010).

[28] J. S. Pedersen, Adv. Colloid Interfac. 70, 171 (1997).

[29] T. G. Mason and M. Y. Lin, Phys. Rev. E 71, 040801 (2005).

[30] M. Stieger, W. Richtering, J. S. Pedersen, and P. Lindner, J. Chem. Phys. 120, 6197 (2004).

[31] B. W. Garner, T. Cai, S. Ghosh, Z. B. Hu, and A. Neogi, Appl. Phys. Express 2, 057001 (2009).

[32] A. Patkowski, E. Gulari, and B. Chu, J. Chem. Phys. 73, 4178 (1980).

[33] M. Sedlak, J. Chem. Phys. 105, 10123 (1996).

[34] S. Q. Zhou and B. Chu, J. Phys. Chem. B 102, 1364 (1998).

[35] K. Fujimoto, Y. Nakajima, M. Kashiwabara, and H. Kawaguchi, Polym. Int. 30, 237 (1993).

[36] G. C. Abade, B. Cichocki, M. L. Ekiel-Jezewska, G. Nägele, and E. Wajnryb, J. Chem. Phys. 132, 014503 (2010).

[37] P. Reuland, B. U. Felderhof, and R. B. Jones, Physica (Amsterdam) 93A, 465 (1978).

[38] D. A. Holden, G. R. Hendrickson, W. J. Lan, L. A. Lyon, and H. S. White, Soft Matter 7, 8035 (2011) 